\title{
Gapped spin liquid states in a one-dimensional Hubbard model with antiferromagnetic exchange interaction
}

\author{
Jianhui Dai ${ }^{1}$, Xiaoyong Feng ${ }^{1}$, Tao Xiang ${ }^{2,3}$, and Yue $\mathrm{Yu}^{2}$ \\ ${ }^{1}$ Zhejiang Institute of Modern Physics, Zhejiang University, Hangzhou 310027, China \\ ${ }^{2}$ Institute of Theoretical Physics, Chinese Academy of Sciences, P O Box 2735, Beijing 100080, China and \\ ${ }^{3}$ Interdisciplinary Center of Theoretical Studies, Chinese Academy of Sciences, P O Box 2735, Beijing 100080, China
}

(Dated: November 5, 2018)

\begin{abstract}
We study the phase diagram of a one-dimensional extended Hubbard model with antiferromagnetic exchange interaction analytically and numerically. The bosonization and transfer-matrix renormalization group methods are used in the corresponding coupling regimes. At half-filling, the system is a Mott insulator with a finite spin excitation gap if the on-site Coulomb repulsion is fairly smaller than the antiferromagnetic exchange $J$. This Mott-insulator is characterized by the bond-chargedensity-wave order or spontaneously dimerization. In the weak-coupling regime where the spincharge separation holds approximately, the critical point separating the gapless and gapped spin liquid phases is $U_{c} \sim J / 2$. However, as $J$ increases, the spin-charge couplings become important and the critical point $U_{c}$ is significantly suppressed and eventually tends to zero as $J \rightarrow \infty$. Away from half-filling, the charge gap completely collapses but the spin gap persists.
\end{abstract}

\section{INTRODUCTION}

The notion of spin liquid state was introduced thirty years ago when P. Fazekas and P.W. Anderson first postulated that due to the frustrated antiferromagnetic coupling the Mott insulating state on triangular lattice does not break the spin-rotational symmetry [1]. The discoveries of high temperature superconductivity and other novel correlated many-body phenomena have stimulated wide interest in spin liquids both theoretically and experimentally $[2]$. For correlated electrons in twodimensional $\mathrm{Cu}-\mathrm{O}$ plane, the most two important energy scales are the kinetic energy and the on-site Coulomb repulsion. However, as far as the magnetic properties of a Mott insulator is concerned, the nearest neighbor antiferromagnetic exchange do play an important role 3 . Recently, F.C. Zhang showed that within the Gutzwillar approximation, a quantum phase transition from a Mott insulator to a gossamer superconductor(which was first proposed by R.B. Laughlin[4]) at half-filling can take place as the on-site Coulomb repulsion is reduced [5]. It was further anticipated that away from half-filling, this gossamer superconducting state can evolve smoothly into the resonant-valence-bond (RVB) spin-liquid phase.

A basic model for investigating the RVB or gossamer states is the so-called t-U-J model [3, [5, 6, 7, [8]. It is an extended Hubbard model by explicitly including an antiferromagnetic exchange interaction. While in two dimensions it remains challenging to accurately solve this model, it may be instructive to study the corresponding one-dimensional system.

The one dimensional t-U-J model is described by the following Hamiltonian

$$
\begin{aligned}
H= & -t \sum_{i \sigma}\left(c_{i \sigma}^{\dagger} c_{i+1 \sigma}+\text { h.c. }\right)+U \sum_{i} n_{i \uparrow} n_{i \downarrow} \\
& +J \sum_{i} \mathbf{S}_{i} \cdot \mathbf{S}_{i+1} .
\end{aligned}
$$

This Hamiltonian is not merely a toy model motivated by the corresponding two dimensional system, it is also relevant to quasi-one-dimensional correlated physics. For example, most of the Bechgaard salts (the (TMTSF $)_{2} \mathrm{X}$ family of quasi-one-dimensional conductors) show close proximity of spin-density-wave(SDW), spin-Peierls, ferromagnetic, and superconducting phases by varying pressures $[9]$. To investigate the coexistence of triplet superconductivity and ferromagnetism in a class of quasi-one-dimensional materials, Japaridze et al studied the model for ferromagnetic exchange with easyplane anisotropy in the large-bandwidth limit [10]. As a byproduct, they predicted a transition to the dimerized ordering phase in the case of weak anisotropy by assuming spin-charge separation in the weak-coupling regime. Comparatively, what was less understood is the generic feature of the phase diagram in the U-J plane for the isotropic antiferromagnetic model(10).

At the first glance, the situation with generic $U, J>0$ might be trivial. Because in the atomic limit there is no explicit frustration between the pair-wise interactions $U$ and $J$, the system is simply a Mott insulator with gapless spin excitations. This is in contrast to another well-known one-dimensional model system, i.e., the conventional extended Hubbard model(CEHM) with the on$\operatorname{site}(U)$ and nearest neighbor site $(V)$ Coulomb interactions. In the CEHM, the pair-wise interactions $U$ and $V$ are frustrated explicitly, and in the atomic limit, the system is a charge-density-wave(CDW) insulator for large $V$ and a SDW insulator for large $U$ [1]. However, if the on-site repulsion $U$ is reduced, the virtual hopping processes may affect the spin physics in Mott insulators. For example, the recent studies have shown that even within the CEHM there exists a new phase in between the CDW and SDW phases. The new phase is characterized by the bond-charge-density-wave(BCDW) order or spontaneous dimerization with gapped charge and spin excitations in a narrow window extending from the weak to intermediate coupling regimes 12, 13, 14, 15]. Usually, such an 
insulating phase is caused by the electron-phonon coupling or explicit frustrations, but now it is caused by the electronic correlations only. With this kind of BCDW phase in mind, we are going to examine the phase diagram of the model (1) in order to clarify the competing Mott insulator physics in one dimension if the double occupation is allowed.

In the present work, we study the phase diagram of the half-filled one-dimensional t-U-J model by using analytical and numerical methods, depending on the ratio $J / t$. It is found that the ground state is always a Mott insulator at half filling and a metal away from half filling. However, the spin excitations have been dramatically changed by the exchange term. There is a gapped spin liquid phase characterized by the BCDW order when the exchange interaction is fairly stronger than the Coulomb repulsion at arbitrary filling. The spin gap stems from the interplay between the kinetic energy and the antiferromagnetic exchange interaction. As spin-charge couplings increase and the bandwidth decreases, the gapped spin liquid phase will be suppressed. All these reveal a new scenario for the creation of spin gaps in a single itinerant electron chain with translational and spin rotational symmetries and without explicit frustrations [16].

\section{WEAK-COUPLING THEORY: BOSONIZATION, RENORMALIZATION GROUP EQUATIONS, AND SEMI-CLASSICAL ANALYSIS}

Let us first consider the phase diagram at half filling in the weak-coupling regime $U / t, J / t \ll 1$. In this regime, the bosonization technique may be used reliably 17, 18, 19, 20. We linearize the spectrum and pass it to continuum limit by substituting $a^{-1 / 2} c_{j \sigma} \rightarrow$ $(2 \pi a)^{-1 / 2} \sum_{r=+,-} e^{i r k_{F} x+i r \varphi_{r, \sigma}(x)}$ where $\varphi_{r, \sigma}(x)$ are the right/left-moving bosonic fields. Introducing charge and spin bosonic fields, $\phi_{c, r}=\left(\varphi_{r, \uparrow}+\varphi_{r, \downarrow}\right) / 2, \phi_{s, r}=$ $\left(\varphi_{r, \uparrow}-\varphi_{r, \downarrow}\right) / 2$, respectively, the Hamiltonian density of the bosonized model is then given by $\mathcal{H}=\mathcal{H}_{c}+\mathcal{H}_{s}+\mathcal{H}_{c s}$. Here, the charge and spin sectors are described by

$$
\begin{aligned}
\mathcal{H}_{c}= & \frac{v_{c}}{2 \pi} \sum_{r=+,-}\left(\partial_{x} \phi_{c, r}\right)^{2}+\frac{g_{\rho}}{2 \pi^{2} a^{2}}\left(\partial_{x} \phi_{c,+}\right)\left(\partial_{x} \phi_{c,-}\right) \\
& -\frac{g_{c}}{2 \pi^{2} a^{2}} \cos 2 \phi_{c} \\
\mathcal{H}_{s}= & \frac{v_{s}}{2 \pi} \sum_{r=+,-}\left(\partial_{x} \phi_{s, r}\right)^{2}-\frac{g_{\sigma}}{2 \pi^{2} a^{2}}\left(\partial_{x} \phi_{s,+}\right)\left(\partial_{x} \phi_{s,-}\right) \\
& +\frac{g_{s}}{2 \pi^{2} a^{2}} \cos 2 \phi_{s}
\end{aligned}
$$

with $\phi_{\nu}=\phi_{\nu,+}+\phi_{\nu,-}$ for $\nu=c, s$ respectively. While, the spin-charge coupling part is given by

$$
\begin{aligned}
\mathcal{H}_{c s}= & -\frac{g_{c s}}{2 \pi^{2} a^{2}} \cos 2 \phi_{c} \cos 2 \phi_{s} \\
& -\frac{g_{\rho s}}{2 \pi^{2}}\left(\partial_{x} \phi_{c,+}\right)\left(\partial_{x} \phi_{c,-}\right) \cos 2 \phi_{s} \\
& +\frac{g_{c \sigma}}{2 \pi^{2}}\left(\partial_{x} \phi_{s,+}\right)\left(\partial_{x} \phi_{s,-}\right) \cos 2 \phi_{c}
\end{aligned}
$$

$$
+\quad \frac{g_{\rho \sigma}}{2 \pi^{2}} a^{2}\left(\partial_{x} \phi_{c,+}\right)\left(\partial_{x} \phi_{c,-}\right)\left(\partial_{x} \phi_{s,+}\right)\left(\partial_{x} \phi_{s,-}\right)(4)
$$

Here, in order to compare the t-U-J model with the CEHM, we follow the weak-coupling $g$-ology approach and adopt the notation of the Ref. 14]. In the lowest orders of $U$ and $J$, the scattering matrix elements are $g_{1 \|}=-a J / 2, g_{1 \perp}=a(U-J / 2), g_{2 \|}=a J / 2, g_{2 \perp}=$ $a(U+J / 2), g_{3 \|}=-a J / 2, g_{3 \perp}=a(U+3 J / 2)$, and $g_{4||}=$ $a J / 2, g_{4 \perp}=a(U-3 J / 2)$. The renormalized velocities and Luttinger couplings of charge and spin sectors are $v_{c}=$ $2 t a+\left(g_{4||}+g_{4 \perp}-g_{1||}\right) / 2 \pi, v_{s}=2 t a+\left(g_{4||}-g_{4 \perp}-g_{1||}\right) / 2 \pi$ and $g_{\rho}=g_{2 \perp}+g_{2 \|}-g_{1 \|}, g_{\sigma}=g_{2 \perp}-g_{2 \| \mid}+g_{1 \| \mid}$, respectively. The coupling constants $g_{c}$ and $g_{s}$ denote the amplitude of the backward and the Umklapp scattering of opposite spins, given by $g_{c}=g_{3 \perp}, g_{s}=g_{1 \perp}$ respectively. $g_{\rho s} / g_{\rho \sigma}$ (and $g_{c s} / g_{c \sigma}$ ) come from the backward (and Umklapp) scatterings of the parallel/opposite spins, respectively, given by $g_{\rho s}=g_{\rho \sigma}=g_{c s}=g_{c \sigma}=-J / 2$ to the lowest order in $J$.

The low-energy properties of the one dimensional t-U-J model in the weak-coupling regime depend on the scaling behavior of these coupling constants during the scaling $a \rightarrow a e^{d l}$. Tha is, the coupling constants run in terms of the following one-loop renormalization group(RG) equations

$$
\begin{aligned}
\lambda d g_{\rho} / d l= & +2 g_{c}^{2}+g_{c s}^{2}+g_{s} g_{\rho s} \\
\lambda d g_{c} / d l= & +2 g_{\rho} g_{c}-g_{s} g_{c s}-g_{c s} g_{\rho s} \\
\lambda d g_{s} / d l= & -2 g_{s}^{2}-g_{c} g_{c s}-g_{c s}^{2} \\
\lambda d g_{c s} / d l= & -2 g_{c s}+2 g_{\rho} g_{c s}-4 g_{s} g_{c s} \\
& -2 g_{c} g_{s}-2 g_{c} g_{\rho s}-4 g_{c s} g_{\rho s} \\
\lambda d g_{\rho s} / d l= & -2 g_{\rho s}+2 g_{\rho} g_{s}-4 g_{c} g_{c s} \\
& -4 g_{c s}^{2}-4 g_{s} g_{\rho s}
\end{aligned}
$$

where $\lambda=4 \pi t a$. Notice that the $\mathrm{SU}(2)$ symmetry in the spin sector ensures $g_{\sigma}=g_{s}, g_{c s}=g_{c \sigma}$, and $g_{\rho s}=g_{\rho \sigma}$. From these RG equations, one finds that $g_{\rho}, g_{c}$ and $g_{s}$ are marginal with the scaling dimension 2, while $g_{c s}$ and $g_{\rho s}$ are irrelevant with the scaling dimension 4 . So, we may first neglect $\mathcal{H}_{c s}$, assuming spin-charge separation in the weak-coupling regime. In this case, one obtains the RG flows of $g_{\rho}, g_{c}$ from $\lambda d g_{\rho} / d l=2\left(g_{c}\right)^{2}, \lambda d g_{c} / d l=2 g_{\rho} g_{c}$ and $g_{s}$ from $\lambda d g_{s} / d l=-2\left(g_{s}\right)^{2}$, respectively. Since both $U$ and $J$ are positive and $g_{\rho}(0)=U+3 J / 2>0, g_{c}$ is always relevant and flows to the strong-coupling fixed point $g_{c}(l) \rightarrow \infty$. This indicates the existence of the charge excitation gap at half-filling. The charge gap is given approximately by $\Delta_{c} \approx t\left|g_{c} / \lambda\right|^{\lambda / 2 g_{\rho}}$ when $g_{c} \ll \lambda$. For the spin excitations, $g_{s}$ will flow to 0 if $g_{s}>0$, or to $-\infty$ if $g_{s}<0$ [20]. The latter case occurs when $J>2 U$ and in this case a spin gap is expected to open. The spin gap is given approximately by $\Delta_{s} \approx t \exp \left(\lambda / 2 g_{s}\right)$ when $0<-g_{s} \ll \lambda$.

Now, we examine how the spin-charge coupling part $\mathcal{H}_{c s}$ affects the phase boundary. As $U$ and $J$ increase, the $g_{c s}$ coupling first becomes less irrelevant in terms of the one-loop RG equations (5)-(9). However, because 
$g_{c}$ grows with increasing $l$ and dominates over the other couplings, the charge excitations are always gapful. Below the charge-gap energy scale the $\phi_{c}$ field is locked at $\left\langle\cos 2 \phi_{c}\right\rangle \simeq\left(\Delta_{c} / t\right)^{2\left(1-g_{\rho} / \lambda\right)}$. Thus, we may re-scale the cosine term in spin sector, by introducing $g_{s}^{*}=g_{s}-g_{c s}$. The one-loop RG equation for $g_{s}^{*}$ is obtained simply by $\lambda d g_{s}^{*}(l) / d l=-2\left(g_{s}^{*}(l)\right)^{2}$. This implies that the spin-gap transition line is shifted to $g_{s}^{*}=0$. To obtain the phase transition line, we follow the same strategy introduced in the Ref. 14] and solve eqs.(5)-(9) numerically by looking at which of the couplings $g_{c}, g_{s}$ and $g_{c s}$ becomes relevant, In our case, $g_{c}$ grows with increasing $l$ faster than the others. We stop the integration once $g_{c}$ reaches 1 and calculate $g_{s}^{*}=g_{s}-g_{c s}$. The positive(negative) $g_{s}^{*}$ leads to the spin gapless(gapful) state. Here, the vertex corrections to the scattering matrices are not included. Although they are indeed crucial for the presence of the narrow BCDW phase in the CEHM 14], their effects are limited in the weak-coupling regime only and may be negligible in the t-U-J model. The phase boundary obtained by this way is shown by the solid line in Fig.1.

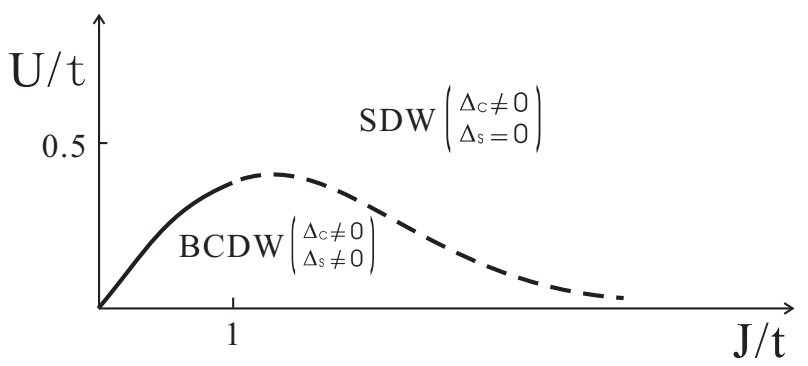

FIG. 1: The ground-state phase diagram of the t-U-J model at half filling. The solid line is the phase boundary determined by numerically solving the RG equations which are valid in the weak-coupling regime. From the intermediate- to strongcoupling regimes, the phase boundary is continued by the dashed line which is qualitatively supported by the numerical data of finite-size small cluster. The low energy correlations are dominated by the SDW and BCDW orders in the gapless and gapped spin liquid phases, respectively.

To characterize the gapped spin liquid phase, we examine via a quasi-classical analysis for four kinds of the order parameters, the SDW, CDW, BCDW, and bond-SDW(BSDW) parameters, defined by $\mathcal{O}_{S D W} \equiv(-1)^{j}\left(n_{j, \uparrow}-n_{j, \downarrow}\right), \mathcal{O}_{C D W} \equiv(-1)^{j} n_{j}$, $\mathcal{O}_{B C D W} \equiv(-1)^{j} \sum_{\sigma}\left(c_{j, \sigma}^{\dagger} c_{j+1, \sigma}+\right.$ h.c. $)$, and $\mathcal{O}_{B S D W} \equiv$ $(-1)^{j}\left(c_{j, \uparrow}^{\dagger} c_{j+1, \uparrow}-c_{j, \downarrow}^{\dagger} c_{j+1, \downarrow}+\right.$ h.c. $)$. Upon bosonization, they are written in terms of bosonic fields as $\mathcal{O}_{C D W}(x) \propto$ $\sin \phi_{c} \cos \phi_{s}, \mathcal{O}_{S D W}(x) \propto \cos \phi_{c} \sin \phi_{s}, \mathcal{O}_{B C D W}(x) \propto$ $\cos \phi_{c} \cos \phi_{s}$, and $\mathcal{O}_{B S D W}(x) \propto \sin \phi_{c} \sin \phi_{s}$ respectively. Neglecting the spatial variations of the fields we focus on the following effective potential

$$
\begin{aligned}
V_{e f f}\left(\phi_{c}, \phi_{s}\right)= & -\tilde{g}_{c} \cos 2 \phi_{c}+\tilde{g}_{s} \cos 2 \phi_{s} \\
& -\tilde{g}_{c s} \cos 2 \phi_{s} \cos 2 \phi_{c} .
\end{aligned}
$$

The couplings $\tilde{g}$ are all effective ones obtained by integrating out high-energy degrees of freedom. As the charge gap exists everywhere at half-filling and is always large than the $\operatorname{spin} \operatorname{gap}\left(\tilde{g}_{c}\right.$ increases faster than $\tilde{g}_{s}$ ), both the BSDW and CDW orders are unfavorable in the ground state. So in the insulating phase, the SDW and BCDW orders compete each other, with the effective potential $V_{S D W}=-\tilde{g}_{c}-\tilde{g}_{s}+\tilde{g}_{c s}$ and $V_{B C D W}=$ $-\tilde{g}_{c}+\tilde{g}_{s}-\tilde{g}_{c s}$ respectively. It is the $\operatorname{SDW}$ (or BCDW) which dominates in the ground state for $\tilde{g}_{c s}<\tilde{g}_{s}$ ( or $\left.\tilde{g}_{c s}>\tilde{g}_{s}\right)$. Therefore, the SDW-BCDW transition line determined by quasi-classical analysis is the same as that of the spin-gap transition line $g_{s}^{*}=0$ given above. In Fig.(1), the deviation of the phase boundary from the straight line $U=2 J$ with increasing $J$ is due to the $g_{c s}$ coupling which in turn enhances SDW order.

\section{INTERMEDIATE AND STRONG-COUPLING REGIMES}

We believe that the existence of the spin gap is a generic feature of the t-U-J model, not limited in the weak-coupling regime only. To further clarify the physics behind the spin-gap, it is interesting to consider the unconstrained t-J model, namely the t-U-J model with $U=0$, in the strong coupling limit $J \gg t$. In this case, one can first bosonize the Heisenberg exchange term and taking the hopping term as a perturbation. In the pure Heisenberg model, the spin excitation is critical but the charge excitation is completely suppressed. Nevertheless, the charge field $\phi_{c}$ should be introduced in the bosonization of the Heisenberg term since charge fluctuations are now allowed. For the pure Heisenberg model, $\phi_{c}$ is pinned to 0 . However, by introducing a small but finite $t$-term, $\phi_{c}$ will become finite. This will reduce the charge gap and at the same time induce effectively a backward scattering term in the spin sector. As a result of this backward scattering, a spin gap will open.

The discussions in the preceding section and the previous paragraph showed the existence of the spin gap in both the weak and strong coupling limits in the unconstrained t-J model. To examine whether this is true in the intermediate coupling regime $J \sim t$, we have calculated numerically the zero-field spin susceptibility using the transfer-matrix renormalization group (TMRG) at half-filling. The TMRG handles directly an infinite lattice system. It does not have any finite lattice size effect and allows a small excitation gap to be accurately determined without invoking the finite size scaling 21]. This is an advantage and the reason for us to use this method here. The error comes mainly from the truncation of basis states in the TMRG iteration. Fig. 2 shows the TMRG result of the uniform spin susceptibility for $J=t$ and $U=0$. In the TMRG calculation, 100 basis states were retained and the maximum truncation error is less than $10^{-5}$. As can be clearly seen from the inset of the figure, the spin susceptibility drops exponentially at low temperatures. This exponential decay of the susceptibility is a direct consequence of the spin gap. 


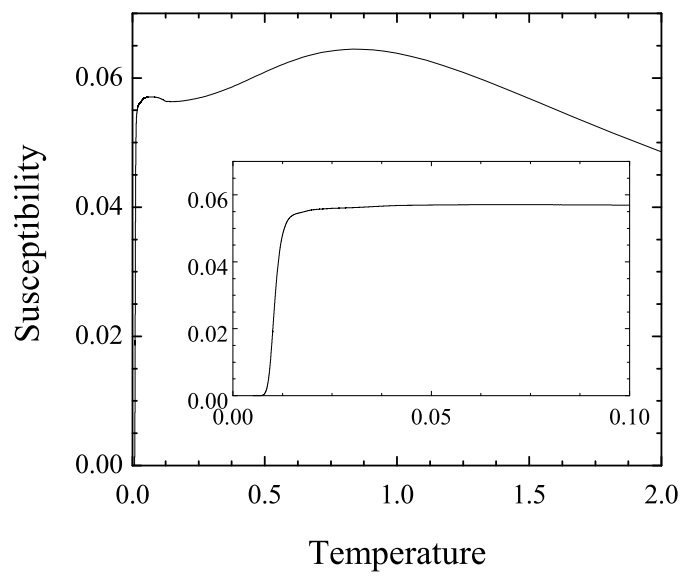

FIG. 2: Zero field magnetic susceptibility of the one dimensional t-U-J model with $J=t=1$ and $U=0$. The inset is an enlarged low temperature plot.

So far, we have established the existence of the gapped spin liquid phase in the weak/strong-coupling regimes and at a special point in the intermediate regime. Now we try to see the generic feature of the phase boundary in the U-J plane. As shown in Fig.1, the phase boundary is very closed to the line $J=2 U$ in the weak-coupling regime, but goes below it as $J$ increases, due to the spincharge couplings. We anticipate that the critical line develops a maximum in the intermediate regime, and then approaches zero gradually as $J$ goes to infinity, see the dashed line in Fig.1.

To support this picture and to estimate the crossing point, we have also studied the spin-gap transition by investigating level-crossings of the excitation spectra [22, 23]. This technique was used to determine the phase boundaries of the CEHM by Nakamura 12 with high accuracy from the numerical data of finite-size clusters. The obtained spin-gap transition line is qualitatively in agreement with Fig.1, the crossing point is about $(1.3,0.35)[24]$.

\section{AWAY FROM HALF-FILLING: WEAK-COUPLING ANALYSIS}

Finally, we briefly address the subsequences after introducing holes into the system. In the weak-coupling regime and away from half filling, the terms resulting from the Umklapp scattering in $\mathcal{H}_{c}$ and $\mathcal{H}_{c s}$ disappear. The other terms are still present but their coupling constants become doping dependent. To the leading order approximation, $g_{\rho}=a\left(U+\frac{3 J}{2} \cos \left(\frac{\pi}{2} \delta\right)\right)$, $g_{s}=a\left(U-\frac{J}{2} \cos \left(\frac{\pi}{2} \delta\right)\right)$ and $g_{\rho s}=-a \frac{J}{2} \cos \left(\frac{\pi}{2} \delta\right)$, where $\delta$ is the doping concentration. Due to the absence of the Umklapp scattering, the charge gap collapses. However, the backward scattering of spin excitations and the subsequent spin gap phase persist. In the limit $U \rightarrow 0$ and $J \rightarrow 0$, the spin gap phase exists when the condition $g_{s}<0$, i.e. $J>2 U \cos ^{-1}\left(\frac{\pi}{2} \delta\right)$, is satisfied. Thus the critical exchange constant increases with increasing doping. In contrast to the half-filling case, the gapped spin state is now metallic. In this Luther-Emery-type state, the CDW and singlet pairing (SP) correlations develop asymptotically as $C_{C D W}(r) \sim a_{1} r^{-2}+a_{2} \cos \left(2 k_{F} r\right) r^{-K_{c}}, C_{S P}(r) \sim$ $a_{3} r^{-1 / K_{c}}$, with $a_{i}$ being constants of order of 1 . Upon doping $\delta>0, K_{c}=\sqrt{\frac{1-g_{\rho} / 2 v_{c}}{1+g_{\rho} / 2 v_{c}}}<1$. Of course, the CDW correlation is the most dominant one in the LutherEmery phase.

\section{SUMMARY}

In summary, we have studied the one-dimensional t-U$\mathrm{J}$ model analytically and numerically in different coupling regimes. At half-filling, the system shows two distinct insulating phases: a gapless spin liquid phase dominated by SDW correlation and a gapped spin liquid phase dominated by BCDW correlation. The suggested phase diagram is plotted in Fig.1. In the weak coupling regime, the phase boundary line is determined by solving the RG equations. This line is anticipated to develop a maximum in the intermediate regime and tends to zero at the strong coupling limit. If $U$ is larger than a critical value (which is estimated about $\sim 0.35 t$, see Ref. [24]), the gapped spin liquid no longer exists, irrespective of the magnitude of $J$. After doping, the gapped spin liquid phase becomes the Luther-Emery phase where the charge gap is completely suppressed while the spin-gap persists.

It is remarkable that in the t-U-J model the spingap behavior resembles to that of the frustrated $t-\mathrm{J}_{1}-\mathrm{J}_{2}$ model [25, 26] but with a quite different mechanism and the charge gap behavior resembles to that of the Hubbard model but with an enhanced magnitude. In the t-U-J model, the spin gap results from the interplay between the antiferromagnetic exchange and the kinetic energy ( the constraint of no double occupancy is released). The BCDW insulator driven by this mechanism should have a large but finite ratio of the charge gap to the spin gap, $\Delta_{c} / \Delta_{s}$. For the band, Kondo and SDW insulators this ratio is unit, $1-1.5$ and $\infty$, respectively. For the conventional spin-Peierls systems, this ratio should be very large. So, the present BCDW phase may be relevant to a class of quasi-one-dimensional Mott-insulators where the charge gaps are only several times larger than the spin ones. Particularly, we expect that the mechanism may be relevant to the Bechgaard salts such as (TMTSF) ${ }_{2} \mathrm{PF}_{6}$ where a transition from the spin-Peierls phase into the SDW phase by changing pressures was observed 27 . 


\section{ACKNOWLEDGEMENT}

We acknowledge useful discussions with S.J. Qin, Y.J. Wang, Z.X. Xu and F.C. Zhang. JD wish to thank L. Yu for the hospitality at the Interdiscipline Center of Theoretical Studies where this work was initiated. This work was supported in part by the National Natural Science Foundation of China and the NSF of Zhejiang Province.
[1] P. Fazekas and P.W. Anderson, Philos. Mag. 30,423(1974).

[2] P. W. Anderson, Science 235, 1196 (1987).

[3] T.C. Hsu, Phys. Rev. B41, 11379(1990).

[4] R.B. Laughlin, cond-mat/0209269

[5] F.C. Zhang, Phys. Rev. Lett. 90, 207002(2003).

[6] Y. Yu, cond-mat/0211131

[7] J.Y. Gan, F.C. Zhang and Z.B. Su, cond-mat/0308398

[8] G. Chapline, Z. Nazario and D. I. Santiago, cond-mat/0309297

[9] D. Jerome, A. Mazaud, M. Ribault, and K. Bechgaard, J. Phys. (Paris)Lett. 41, L95(1980).

[10] G.I. Japaridze and E. Muller-Hartmann, Phys. Rev. B61,9019(2000); C. Dziurzik, G.I. Japaridze, A. Schadchneider and J. Zittartz, cond-mat/0402405

[11] R.A. Bari, Phys. Rev. B3, 2662(1971); J.E. Hirsch, Phys. Rev. Lett 53, 2327(1984); J.W. Cannon and E. Fradkin, Phys. Rev. B41 , 9435(1990); J. Voit, Phys. Rev. B 45, 4027(1992); G.P. Zhang, Phys. Rev. B56, 9189(1997).

[12] M. Nakamura, J. Phys. Soc. Jpn.68,3123(1999); Phys. Rev. B 61, 16377(2000).

[13] P. Sengupta, A.W. Sandvik, and D.K. Campbell, Phys. Rev. B 65, 155113(2002).

[14] M. Tsuchiizu and A. Furusaki, Phys. Rev. Lett. 88, 056402 (2002).

[15] It was found earlier that when the Hubbard repulsion is strong there is an instability both in the CDW and SDW phases of the half-filled CEHM within slave-boson frame- work, indicating the existence of a new and intermediate phase, see S. Caprara, M. Avignon and D. D. Sarma, Int. J. Mod. Phys. B, 11, 2057(1997).

[16] M. Oshikawa, Phys. Rev. Lett. 84, 1535(2000).

[17] V.J. Emery, in Highly Conducting One-Dimensional Solids, edited by J. Devereese et.at (Plenum, New York,1979),p.247.

[18] J. Solyom, Adv. Phys. 28, 201(1979).

[19] J. Voit, Rep. Prog. Phys. 57, 977(1994).

[20] A.O. Gogolin, A.A. Nersesyan, and A.M. Tsvelik, Bosonization and Strongly Correlated Systems, Cambridge University Press, NY, 1998.

[21] X. Wang and T. Xiang, in Density-Matrix Renormalization Group: a New Numerical Method in Physics(Springer,1999), chap.6, p.149.

[22] R. Julien and F.D.M. Haldane, Bull. Am. Phys. Soc. 28,34(1983).

[23] K. Okamoto and K. Nomura, Phys. Lett. A169, 433(1992).

[24] X. Feng, Z. Xu, and J. Dai, unpublished, 2004.

[25] M. Imada, J. Phys. Soc. Jpn. 60,1877(1991)); Physca C185-189, 1421(1991); Prog. Theor. Phys. Supp. 133, 203(1993).

[26] M. Ogata, M.U. Luchini and T.M. Rice, Phys. Rev. B 44, 12083(1991).

[27] D. Jerome, Science 252,1509(1991). 